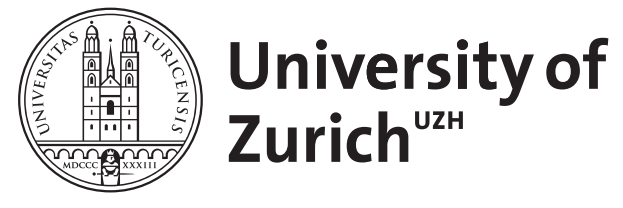

\title{
Anthropologie und Ethik des Enhancements
}

Heilinger, J C

\begin{abstract}
Biotechnologische Eingriffe in den menschlichen Organismus (genetisch, psychopharmazeutisch oder technisch) können die körperliche oder geistige Leistungsfähigkeit von Menschen steigern und das menschliche Erleben gezielt verändern, auch über ein "normales" oder "natürliches" Maß hinaus. Der Autor untersucht die ethische Debatte zu diesem Thema, insbesondere Argumente, die ausgehend von einem normativen Begriff vom Menschen bestimmte Eingriffe verbieten oder erlauben wollen. Er entwickelt ein integratives, anthroponomes Modell einer signifikanten "Kartierung" des Begriffs Mensch, das es erlauben soll, den Begriff in normativer Hinsicht möglichst stark zu machen. Dennoch sind anthropologische Argumente - angesichts von Risiko- und Gerechtigkeitserwägungen - lediglich ein Element einer umfassenderen Ethik des Enhancements.
\end{abstract}

Posted at the Zurich Open Repository and Archive, University of Zurich

ZORA URL: https://doi.org/10.5167/uzh-41239

Monograph

Published Version

Originally published at:

Heilinger, J C (2010). Anthropologie und Ethik des Enhancements. Berlin: De Gruyter. 


\section{Inhalt}

Einleitung: Anthropologische Elemente einer Ethik

des Enhancements .......................... 1

Übersicht über die Arbeit $\ldots \ldots \ldots \ldots \ldots \ldots \ldots \ldots \ldots$

I. Die anthropologische Relevanz von Human Enhancement

1. Die Forschungsfrage und ihre disziplinäre Einbindung . . 15

1.1. Anthropologie ...................... 17

1.2. Meta-Ethik ........................ 19

1.3. Normative Ethik . . . . . . . . . . . . . . . . . . 20

1.4. Angewandte Ethik .................... 23

2. Biotechnologische Interventionen $\ldots \ldots \ldots \ldots \ldots .24$

2.1. Technische Eingriffe oder Erweiterungen ......... 26

2.2. Genetisches Engineering . ................ 27

2.3. Biologische Prozessoptimierung im Organismus ...... 30

3. „Alte“ und „neue“ Interventionen:

Kontinuität der Mittel und Ziele . . . . . . . . . . . 33

3.1. Kontinuität der Mittel ... . . . . . . . . . . . . 34

3.2. Kontinuität der Ziele .................... 38

4. Das Projekt einer deskriptiv-normativen begrifflichen

Selbstauslegung ..................... 42

4.1. Begriffliche Selbstauslegung des Menschen ......... . 42

4.2. Naturalistische Erklärungen als Herausforderung für die Anthropologie . . . . . . . . . . . . . . . . 46

4.3. Jenseits des Naturalismus: Was soll der Mensch sein? .... 52 
II. Was ist Enhancement? Begriffsanalysen und Definitionen

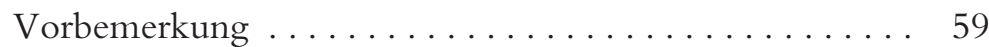

5. Gesundheit und Krankheit ................ 62

5.1. Ein „objektiver“ Begriff von Gesundheit und Krankheit 63

5.2. Ein ,subjektiver" Begriff von Gesundheit und Krankheit 65

5.3. Ein ,relationaler“ Begriff von Gesundheit und Krankheit 66

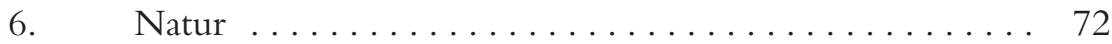

6.1. Schwierigkeiten der Begriffsbestimmung ........ 73

6.2. Vielfältige Gegenbegriffe zur „Natur“ .......... 74

6.3. Binnendifferenzierungen des Naturbegriffs ........ 76

7. Natur des Menschen ..................... 82

7.1. Das biologische Kriterium der Spezieszugehörigkeit . . . . 83

7.2. Die ,typisch“ menschliche Lebensform .......... 85

7.3. Geworden und Gemacht .............. 88

7.4. Begriffliche Schwierigkeiten: „Natur“ und „Natur des Menschen“ . . . . . . . . . . . . . . . . 89

8. Eine dynamische Minimaldefinition von Enhancement .. 91

8.1. Eine dynamische Minimaldefinition ............ 91

8.2. Einschätzung der dynamischen Minimaldefinition ..... 96

III. Eine integrative Kritik der Enhancementdebatte

Vorbemerkung ........................ 103

9. Hoffnungen und Verheißungen ............. 105

9.1. Transhumanisten und andere Befürworter weit reichender Enhancements . . . . . . . . . . . . . 105

9.2. Drei Thesen des Transhumanismus . . . . . . . . . 117

9.3. „Entmystifizierung“ des Transhumanismus ......... 122

10. Festhalten am status quo . . . . . . . . . . . . 125

10.1. Der Report des US-amerikanischen President's Council on Bioethics . . . . . . . . . . . . . . . . . . . . 125

10.2. Vier ,essential sources of concern“ . . . . . . . . . 130

10.3. Eine ideologische Position . . . . . . . . . . . 142 
11. Abwägen von Chancen und Risiken ........... 145

11.1. Ansatz und Methode der Studie Intervening in the Brain . . 145

11.2. Die Verantwortung der Politik . . . . . . . . . . . . . 147

11.3. Der prinzipienethische Ansatz: Principles of Biomedical Ethics 151

11.4. Eine prinzipienethische Einschätzung biotechnologischer Enhancements . . . . . . . . . . . . . . . . . . . . 156

12. Gefahren der Einseitigkeit - ein integrativer Ansatz . . . 165

\section{Anthropologie und anthroponome Ethik}

Vorbemerkung . . . . . . . . . . . . . . . . . . . 177

13. Voraussetzungen anthropologischer Argumente:

Begriff und Normativität . . . . . . . . . . . . . 180

13.1. Begriff und Einzelding . . . . . . . . . . . . 180

13.2. Normativität und Normalität im außermoralischen Sinne 184

13.3. Normativität von Begriffen im moralischen Sinne ..... 188

14. Kritik simpler anthropologischer Argumente ........ 191

14.1. Die Attraktivität normativer Natürlichkeitsvorstellungen 192

14.2. Die Kritik an normativen Natürlichkeitsvorstellungen . . 194

15. Das Ziel: Eine signifikante Kartierung des Begriffs „Mensch“ . . . . . . . . . . . . . . . . . . . . . 199

15.1. Eine signifikante Kartierung des Begriffs „Mensch“ . . . . 199

15.2. „thick moral concepts“ - Die Verquickung von Fakten und Wertungen ...................... 204

16. Die quasi-demokratische Begründung anthropologischer Argumente . . . . . . . . . . . . . . . . . . . 207

16.1. Öffentlichkeit als Bedingung . . . . . . . . . . . . 209

16.2. Wechselseitige Information . . . . . . . . . . . . . 213

16.3. Partizipation und Konsens . . . . . . . . . . . . 214 
17. Der Inhalt anthropologischer Argumente . . . . . . . 220

17.1. Die vier signifikanten Komponenten (K1-K4) . . . . . . 223

17.2. Anthropologische Bedingungen für moralisch legitime Enhancements (B1 und B2) . . . . . . . . . . . . . 240

18. Einwände gegen meinen Vorschlag der Begründung anthropologischer Argumente . . . . . . . . . . . . . . 246

18.1. Essentialismus und die „Wahrheit“ der Selbstbestimmung 247

18.2. Die Praktikabilität der quasi-demokratischen normativen Selbstbestimmung . . . . . . . . . . . . . . . 252

18.2.1. Bestimmung des Menschen als Aufgabe .......... 252

18.2.2. Formen quasi-demokratischer Selbstbestimmung . . . . . 254

18.2.3. Erschließende vs. abschließende Verständigung . . . . . . 257

18.2.4. Fünf Einschränkungen des idealen Diskurses: ein realistischeres Modell . . . . . . . . . . . . . . . . . 257

18.3. Kollektiver Irrtum . . . . . . . . . . . . . . . . . . 260

18.4. Anthroponome Anthropologie .............. 263

19. Die Anwendung anthropologischer Argumente . . . . . 266

19.1. Lebensverlängerung . . . . . . . . . . . . . . 268

19.2. Technisierungen des menschlichen Organismus: das Hinzufügen neuer Sinne . . . . . . . . . . . . . 276

19.3. Biologische Prozessoptimierung: Cognitive Enhancement 280

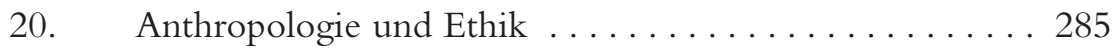

Bibliographie . . . . . . . . . . . . . . . . . . . . 290

Personenregister . . . . . . . . . . . . . . . . . . . 307

Sachregister ....................... 310 


\section{Einleitung: Anthropologische Elemente einer Ethik des Enhancements}

Mit neuen Biotechnologien können Menschen nicht nur ihre Umwelt, sondern zunehmend auch sich selbst immer weitreichender und präziser nach eigenen Vorstellungen verändern. Verlängerungen der gesunden Lebensspanne, psychopharmazeutische Stimmungsaufhellungen und kognitive Leistungssteigerungen, Technisierungen des Gehirns bis hin zum Hinzufügen neuer Sinne werden möglich. Solche Eingriffe, die potentiell weitreichende Veränderungen der menschlichen Lebensform mit sich bringen, werden gegenwärtig als Human Enhancements - „Verbesserungen des Menschen“ - diskutiert. Die „Verbesserungen“ betreffen möglicherweise nicht nur einzelne Individuen in körperlicher oder mentaler Hinsicht, sondern auch das Zusammenleben von Menschen, die Kommunikation zwischen Individuen sowie das Verständnis dessen, was es heißt, Mensch, Person oder Träger moralischer Rechte und Pflichten zu sein. Die Veränderungen, die mit dem Einsatz von Enhancements einhergehen könnten, sind dramatisch. Die menschliche Lebensform könnte anders werden, als sie uns gegenwärtig vertraut ist. Angesichts dieser Möglichkeit stellt sich die Aufgabe einer Verständigung darüber, welche menschlichen Eigenschaften als besonders bedeutsam angesehen werden und deshalb als bewahrenswert gelten sollen. Damit aber wird der - zeitliche und logische - Vorrang der biologisch-natürlichen vor der kulturellen Dimension der menschlichen Existenz zumindest teilweise umgekehrt: Ein kultureller Verständigungsprozess liefert wichtige Voraussetzungen für die biologisch-natürlichen Grundlagen der menschlichen Existenz.

Neben einer Auseinandersetzung mit der problematischen Frage nach dem normativen Gehalt des menschlichen Selbstverständnisses werden in der aktuellen Debatte über die Bewertung von Enhancements vor allem praktische Fragen des Umgangs mit medizinischen und finanziellen Ressourcen aufgeworfen, die für die Durchführung der Eingriffe nötig sind, aber nicht unbegrenzt zur Verfügung stehen. Darüber hinaus ist angesichts des unvollständigen menschlichen Wissens - auch ein Verfehlen der angestrebten Ziele möglich; Risiken sind nicht vollständig 
kalkulierbar und Nebenwirkungen durch den Umgang mit den neuen Technologien, die in den hyperkomplexen menschlichen Organismus eingreifen, können nicht ausgeschlossen werden. Außerdem stellt sich die Frage, inwieweit die Enhancement-Eingriffe tatsächlich auf freien Entscheidungen der Individuen basieren oder ob sie vom subtilen gesellschaftlichen Druck, der etwa in einer kompetitiven Leistungsgesellschaft herrscht, beeinflusst sind. Wie kann, all diese Aspekte berücksichtigend, eine angemessene ethische Bewertung der biotechnologischen Veränderungen, die Menschen an sich vornehmen können, aussehen?

Diese Frage wird gegenwärtig nicht nur in akademischen Fachkreisen, sondern unter reger Anteilnahme der Öffentlichkeit diskutiert. Den großen Hoffnungen, die Menschen in die Biotechnologien setzen, wenn es um die Verbesserung des menschlichen Lebens geht, stehen extreme Ängste vor einem Ende der uns vertrauten menschlichen Lebensform gegenüber. Können wir mithilfe der Biotechnologien dauerhaft Krankheiten überwinden, die gesunde und aktive Lebensspanne verlängern und uns dabei fast permanent glücklich fühlen? Wird der Mensch zu einem von Technik geprägten, steuerbaren perfekten Wesen? Lässt sich die menschliche Lebensform tatsächlich vollständig auf wissenschaftlich erforschbare Abläufe reduzieren und damit auch manipulieren? Diese Fragen zeigen, warum die ethische Diskussion über den Einsatz von Human Enhancements aktuell so viel Aufmerksamkeit erfährt: Das Menschsein insgesamt scheint auf dem Spiel zu stehen.

Man kann bei einer ethischen Diskussion versuchen, auf vertraute Instrumente der ethischen Analyse von Handlungsoptionen zurückzugreifen. Aus utilitaristischer Perspektive etwa wird gefragt, ob mithilfe der angestrebten Veränderungen von Menschen ein näher zu definierender Nutzen oder das Wohlbefinden gesteigert werden können. Deontologisch beeinflusste Ethiker diskutieren, ob einige Interventionen in den menschlichen Organismus kategorisch verboten sein sollten oder ob sich eventuell eine näher zu spezifizierende Verpflichtung zum Einsatz der neuen Technologien erkennen lässt. Im Kontext biomedizinischer Ethik und Technikfolgenabschätzung bezieht man sich auf etablierte moralische Prinzipien - etwa Respekt vor autonomen Entscheidungen, Vermeidung von Schaden, Begrenzung von Risiken, gerechte Verteilung knapper Güter. Die genannten etablierten Analyse- und Bewertungskriterien dominieren in der aktuellen Diskussion über Human Enhancement, die sich mittlerweile als ein neues Arbeitsgebiet der biomedi- 
zinischen Ethik etabliert hat. ${ }^{1}$ Doch angesichts einer möglichen Veränderung von Menschen - einer Erweiterung ihrer kognitiven Fähigkeiten oder ihrer Sinneskapazitäten, einer massiven Ausdehnung der gesunden Lebensspanne oder der Kontrolle über Stimmungen und Wohlbefinden greift die standardmäßige Anwendung etablierter moralischer Bewertungsmaßstäbe zu kurz. Wie nämlich sähe etwa eine utilitaristische Folgenabschätzung (etwa im Sinne von Benthams hedonistischem Handlungskonsequentialismus) aus, wenn gerade die Lustempfindungsfähigkeit von Menschen modifiziert werden würde? Wie sind Risiken einzuschätzen, wenn veränderte Menschen möglicherweise völlig anders empfinden und damit andere als die gegenwärtig dominierenden Bewertungsmaßstäbe anlegen würden? Wie kann eine in der Struktur der Vernunft begründete deontologische Ethik (etwa im Sinne Kants) als Maßstab der Bewertung von Veränderungen gelten, wenn womöglich die Struktur der Vernunft Gegenstand der Veränderungen ist?

Hier zeigt sich die fundamentale Herausforderung, die auch die ethische Beurteilung erschwert: Weil letztlich die ganze menschliche Lebensform eine andere sein könnte, ist die einfache Anwendung bereits etablierter moralischer Maßstäbe möglicherweise unpassend. Die Frage, ob und wie Menschen sich verändern dürfen und sollen, ist von besonderer Art und nicht einfach analog zu anderen Problemen der angewandten Ethik abzuhandeln. Die genannten Bewertungsmaßstäbe bedürfen daher zur Beurteilung der Enhancement-Problematik einer besonderen theoretischen Begründung und inhaltlichen Ergänzung.

Bei näherer Betrachtung der Debatten über Human Enhancement zeigt sich, dass ein bestimmter Typ von Argumenten hier häufiger in Erscheinung tritt als in anderen Bereichen der biomedizinethischen Bewertung technologischer Handlungsoptionen, nämlich ,,anthropologische Argumente“. Darunter verstehe ich Bewertungen von Handlungsoptionen, die von einem bestimmten normativen Verständnis des Menschen ausgehen. Manche Handlungen stehen, so wird dann behauptet, in einem Widerspruch zu dem, wie der Mensch ist oder wie er sein soll. Auch wenn die direkte Berufung auf eine moralisch wertvolle, feststehende „Natur des Menschen“ argumentative und konzeptuelle Defizite aufweist und zumeist als Tabu und als Diskussionsstopper von Skeptikern der neuen Technologien vorgebracht wird, leuchtet es ein,

1 Zur Übersicht über die aktuelle Debatte vgl. die Textsammlungen von Gordijn/ Chadwick 2008, Savulescu/Bostrom 2009, Schöne-Seifert et al. 2008 nach dem Vorreiter Parens 2000. 
dass die Frage, was der Mensch ist oder was er sein soll, eine wichtige Rolle angesichts der Bewertung möglicher - und wohl auch unvermeidlicher (Baylis/Robert 2004) - Veränderungen von Menschen spielt. Der Zukunftsforscher Ray Kurzweil schrieb kurz vor Beginn des 21. Jahrhunderts: „The primary political and philosophical issue of the next century will be the definition of who we are." (Kurzweil 2000, 2). Er verweist damit auf die politische Dimension der philosophisch-anthropologischen Frage nach dem Menschen. Die Frage, was der Mensch ist, was er sein soll oder welche „menschlichen“ Eigenschaften als signifikant und bewahrenswert ausgezeichnet werden sollen, ist daher Gegenstand der aktuellen Debatten, deren Antworten deskriptiv und normativ gehaltvoll sein müssen.

Die vorliegende Arbeit wendet sich anthropologischen Argumenten zu und versucht, ihre Rolle im Kontext einer ethischen Analyse möglicher Human Enhancements zu bestimmen. Damit wird das fundamentale systematische Problem des Zusammenhangs von Anthropologie und Ethik anhand eines konkreten Anwendungsfalls diskutiert. Die Reichweite des problematischen Verhältnisses von Anthropologie und Ethik geht jedoch über die Enhancement-Debatte hinaus. Immer wenn die Position vertreten wird, dass Menschen einen besonderen normativen Status haben - etwa besonders wertvolle Eigenschaften, intrinsische Rechte oder eine spezifische Würde - treten normative anthropologische Argumente auf.

Anthropologische Argumente sind jedoch kontrovers. So häufig sie in den Debatten vorgebracht werden, so umstritten ist ihre Tragfähigkeit. Daher werde ich „schwache“, nicht hinreichend begründete, von „starken“, tragfähigen anthropologischen Argumenten unterscheiden. Daran anschließend werde ich ein konkretes Modell für die angemessene Begründung (durch die Angabe eines Verfahrens) und inhaltliche Bestimmung (in Form von vier Komponenten) tragfähiger anthropologischer Argumente vorstellen. Damit soll den alternativen ethischen Bewertungen von Human Enhancement eine möglichst tragfähige anthropologische Fundierung und Ergänzung an die Seite gestellt werden.

Einen direkten Zugang zur Erkenntnis dessen, was der Mensch wahrhaft ist oder sein soll, halte ich jedoch für unmöglich. Daher gehe ich davon aus, dass zur Beantwortung der Frage, was der Mensch ist und was er sein soll, kein anderer Weg besteht, als das deskriptiv-normative Verständnis des Begriffs „Mensch“ (das den Kern anthropologischer Argumente darstellt) anthroponom, also durch Menschen selbst, zu bestimmen. Im Folgenden geht es mir also darum, ausgehend vom Impuls 
der Enhancement-Debatte einen Beitrag zur Bestimmung des menschlichen Selbstverständnisses unter den Bedingungen der Gegenwart und damit zu einer anthroponomen Ethik zu leisten. Zentrale Merkmale dieses Ansatzes sind - aus einer aufklärerischen Tradition - die kritische Skepsis gegenüber vermeintlich ewigen oder feststehenden Wahrheiten, sowie - aus einer Tradition, die humanistisch genannt werden kann - die Betonung der menschlichen Verantwortlichkeit für das eigene Leben als Individuum und auch als Menschheit. Außerdem werden - gemäß eines pragmatistischen Ansatzes - normative Regeln und Prinzipien als fallibel und entwicklungsfähig konzipiert, so dass es auch in ethischen Systemen Verbesserungen, ja Fortschritt, geben kann.

Diese drei Merkmale machen deutlich, dass das hier vorgeschlagene Modell für eine angemessene Begründung anthropologischer Argumente keine ewige Wahrheit oder Gültigkeit beanspruchen kann. Mein Anspruch ist bescheidener. Die in dieser Arbeit in Auseinandersetzung mit der aktuellen bioethischen Debatte über Human Enhancement skizzierte Methode und inhaltliche Bestimmung anthropologischer Argumente versteht sich als ein Zwischenergebnis, das auf der Grundlage der spezifisch westlichen Tradition ethischer Theoriebildung als möglicher Ausgangspunkt für weitere Bestimmungen und Präzisierungen der Antwort auf die Frage beitragen soll, wie wir als Menschen uns angemessen - i.e. signifikant - selbst bestimmen und normativ bewerten sollten.

Die Unvollständigkeit und Unfertigkeit der hier vorgelegten Skizze entspricht dem untersuchten Gegenstand. Der Versuch einer abgeschlossenen Darstellung der begrifflichen Selbstauslegung des Menschen wäre dahingegen unangemessen: Ein solcher Versuch würde den notwendig aktiven, geradezu performativen und kreativen Charakter des erschließenden Prozesses menschlicher Selbstauslegung und Selbstbestimmung verkennen. Es ist allein dieser aktive Prozess der deskriptiv-normativen begrifflichen Selbstauslegung des Menschen, der - wenn überhaupt irgendetwas - eine tragfähige Grundlage für anthropologische Argumente darstellen kann, wie sie etwa in den Debatten über Human Enhancement herangezogen werden.

Der Begriff „Mensch“ soll im Kontext dieser Arbeit nicht zu eng verstanden werden. Wie ich in meiner kritischen Darstellung alternativer normativer anthropologischer Argumente darlegen werde, ist ein biologischer oder gar rein genetisch bestimmter Begriff „Mensch“ zu eng. Biologische Grundlagen stellen allenfalls eine Komponente eines signifikanten Begriffs vom Menschen dar. Damit kann nicht grundsätzlich 
ausgeschlossen werden, dass auch Wesen, die nicht in biologischer Hinsicht eindeutig als homo sapiens zu bestimmen sind, unter den hier zu entwickelnden Begriff fallen. Die vermeintlich präzise, enge Speziesgrenze stellt jedenfalls keine feststehende moralische Grenze dar.

Die hier vorgestellten Überlegungen nehmen also die aktuelle Debatte über Human Enhancement zum Ausgangspunkt und zugleich als Anwendungsbeispiel für eine $\mathrm{zu}$ entwickelnde deskriptiv-normative Bestimmung des Begriffs „Mensch“, die als regulative Idee zu einer Bewertung von Handlungsoptionen beitragen soll. Mit der im Folgenden von mir versuchten Integration anthropologischer, angewandt ethischer, meta-ethischer und normativer Aspekte riskiere ich jedoch, manchen Erwartungen nicht gerecht werden zu können. Denjenigen, die praxisorientiert an einer konkreten Diskussion spezifischer EnhancementTechnologien und einer diesbezüglichen Handlungsempfehlung interessiert sind (z.B.: Soll es erlaubt werden, dass Menschen Technik X anwenden?), wird meine Arbeit möglicherweise zu theoretisch und zu wenig konkret erscheinen. Denjenigen, die von einem theoretischen Interesse motiviert einen Beitrag zur normativen oder zur Meta-Ethik suchen, wird dahingegen mein Beitrag vielleicht zu konkret und nicht genügend theoretisch vorkommen. Denjenigen, die aus der Tradition der Philosophischen Anthropologie stammen, mag meine Arbeit vielleicht zu moralphilosophisch erscheinen; vielleicht wird dann der Vorwurf erhoben, dass die bereits gewonnenen Einsichten dieser Denkrichtung nicht hinreichend aufgegriffen werden. Denjenigen, die von einem genuin ethischen Interesse geleitet sind, mag die Betonung der anthropologischen Dimension zu weit gehen. Trotz dieser Risiken erscheint mir der Versuch einer Verbindung der genannten philosophischen Teildisziplinen - Anthropologie, angewandte, normative und Meta-Ethik notwendig, um zu einer umfassenden Bewertung des Phänomens Human Enhancement zu gelangen, die nicht nur punktuell bei der Beurteilung einer bestimmten Technik von Bedeutung ist, sondern darüber hinaus als relevanter Beitrag zur möglichst guten Gestaltung des Zusammenlebens von Individuen verstanden werden kann. In dieser Absicht ergänzt eine solche umfassende Bewertung möglicher Enhancements die eingangs erwähnte übliche Diskussion, wie sie auch anhand zahlreicher anderer bioethischer Probleme geführt wird, um den Hintergrund tragfähiger anthropologischer Argumente.

Wenn es mir mit meinen Überlegungen gelingen sollte, die Leserinnen zu überzeugen, dass anthropologische Argumente in der ethischen Beurteilung der möglichen Anwendung von Enhancement-Technolo- 
gien eine wichtige (wenn auch subsidiäre und keineswegs ausschließliche) Rolle spielen, hätte ich mein erstes Ziel erreicht. Wenn darüber hinaus auch mein methodischer und inhaltlicher Vorschlag als ein valider Ausgangspunkt (nicht als der einzig mögliche) für das Verständnis der Rolle anthropologischer Argumente akzeptiert werden würde, hätte ich auch mein zweites Ziel erreicht. Ein drittes Ziel wäre schließlich erreicht, wenn sich Leser durch meine Darstellung aufgefordert fühlen würden, Kritik zu äußern, Veränderungen und Verbesserungen an dem hier vorgestellten Modell vorzuschlagen, und damit in den Prozess der begrifflichen Selbstauslegung von Menschen einzusteigen, zu dem ich einen Vorschlag machen möchte. Schließlich ist der Gegenstand vorliegender Arbeit die Tatsache, dass Menschen darüber nachdenken und sich darüber verständigen, wie sie sich unter den Bedingungen einer sich stets verändernden Gegenwart selbst verstehen und beschreiben; und sich außerdem darüber verständigen, welche Eigenschaften und Merkmale sie an sich selbst als signifikant und bewahrenswert auszeichnen wollen.

Die Bedeutung der anthropologischen und ,anthroponomen“ Selbstbestimmung ist kaum zu unterschätzen. Die Antworten auf die Frage, wer wir sind und wie wir sein wollen, wirken nachhaltig in allen Sphären des menschlichen Lebens und beeinflussen die verschiedenen ethischen Standards, die wir zur Bewertung von Handlungsoptionen anlegen können. Daher rede ich - in einem zweifachen Sinne - von den anthropologischen Elementen der Ethik des Enhancements. Die Anthropologie ist zum einen ein Element neben anderen in der Debatte über Human Enhancement, das die etablierte Debatte mit ihrer Fokussierung auf Risiko- und und Gerechtigkeitserwägungen sowie ihrer Diskussion des informed consent um eine weitere Perspektive ergänzt. Anthropologische Argumente stehen damit - einerseits - paritätisch auf einer Ebene mit anderen Argumenten.

Zum anderen ist die anthropologische Dimension elementar im Sinne von fundamental, da die im Rahmen der Anthropologie vorgenommenen basalen Bestimmungen in Form von menschlichen Wertungen und Handlungen weitreichende Wirkung entfalten. Hiermit ist die Stellung anthropologischer Argumente gegenüber den anderen ethischen Argumenten nicht mehr paritätisch, sondern - andererseits - gewissermaßen subsidiär oder fundierend. Anthropologische Elemente sind dann als „Spurenelemente“ in den anderen Argumentklassen zu finden.

Diese doppelte Relevanz anthropologischer Elemente im Kontext einer ethischen Diskussion von Human Enhancement-Technologien soll im Verlauf der Arbeit entfaltet werden. 
$\mathrm{Zu}$ Beginn (Teil I.) werde ich die verschiedenen Dimensionen der Enhancement-Problematik und ihre Relevanz beleuchten. Daraufhin (Teil II.) werden begriffliche Schwierigkeiten in diesem Zusammenhang diskutiert; anschließend (Teil III.) wende ich mich kritisch der aktuell geführten Debatte zu. Abschließend wird (Teil IV.) ein Modell vorgestellt, dass die anthropologischen Elemente der ethischen Diskussion von Enhancement expliziert. Damit werden insgesamt Fragen berührt, die auch über den begrenzten Horizont der Enhancement-Diskussion hinausweisen. Sie betreffen die Begründung von Normativität, die begriffliche Selbstauslegung von Menschen und die Aufgabe einer angemessenen Gestaltung des Zusammenlebens in der Welt. 


\section{Übersicht über die Arbeit}

Der erste Teil präsentiert die Forschungsfrage nach der Rolle anthropologischer Argumente bei der ethischen Bewertung der Möglichkeit, Menschen mithilfe biotechnologischer Interventionen zu „verbessern“. Dazu wird zunächst (Kapitel 1) die Forschungsfrage selbst in ihrer disziplinären Einbettung erläutert. Daran anschließend werden (Kapitel 2) die Technologien, die den konkreten Anlass für die anthropologischen Reflexionen darstellen, systematisch vorgestellt. Dabei zeigt sich (Kapitel 3), dass auch die neuen Technologien in einer Kontinuität zu ,alten“ Mitteln und Zielen menschlichen Handelns stehen. Zum Abschluss des einleitenden Teils wird (Kapitel 4) der Rahmen der vorliegenden Arbeit abgesteckt: Die hier unternommene Bestimmung anthropologischer Argumente in einem spezifischen bioethischen Kontext ist Teil des größeren Projekts der begrifflichen Selbstauslegung des Menschen, das sich in der Gegenwart aus naturwissenschaftlichen und introspektiven, aus normativen und deskriptiven Anteilen zusammensetzen muss.

Der zweite Teil wendet sich begrifflichen Klärungen zu. Insbesondere der Begriff „Enhancement“ selbst ist notorisch unklar. Auf der Suche nach einer möglichst adäquaten Bestimmung des Begriffs „Enhancement" werden (Kapitel 5) die im Hintergrund der Begriffsbestimmung wirksamen Konzepte von „Gesundheit“ und „Krankheit“ problematisiert. Außerdem werden Schwierigkeiten bei der Verwendung der Begriffe „Natur“ (Kapitel 6) und „Natur des Menschen“ (Kapitel 7) nachgewiesen. Vor dem Hintergrund dieser konzeptuellen Probleme zeigt sich (Kapitel 8), dass eine angemessene Definition von „Enhancement" dynamisch und minimal sein muss; das heißt, dass sie sich mit Blick auf gegebene Kontexte verändert und lediglich auf einen Teilbereich von Enhancements fokussiert, der im Rahmen der Enhancement-Debatte als ethisch relevantes Problem verstanden wird. Damit wird deutlich, dass nicht alle Enhancements per se aus bioethischer Sicht problematisch sind, sondern nur bestimmte Formen weit reichender biotechnologischer Eingriffe den Gegenstandsbereich der bioethischen Enhancement-Debatte konstituieren.

Im dritten Teil soll die Notwendigkeit einer integrativen, anthropologischen Diskussion der Enhancement-Problematik erwiesen werden. 
Dazu wird kritisch die aktuelle bioethische Debatte exemplarisch nachgezeichnet. Hier stehen sich (Kapitel 9) fortschrittsoptimistische, doch übermäßig wissenschaftsgläubige Positionen wie die der sogenannten „Transhumanisten“ auf der einen, sowie (Kapitel 10) skeptische Positionen auf der anderen Seite gegenüber. Letztere, die am Fall des USamerikanischen President's Council on Bioethics vorgestellt werden, zeigen manchmal fundamentalistische Züge. Zwischen diesen beiden extremen Positionen wird (Kapitel 11) der Versuch unternommen, ergebnisoffen und unter Anwendung ökonomischer und gegenwärtig akzeptierter ethischer Standardbewertungen zu einer Bewertung der neuen Technologien und ihrer möglichen Enhancement-Anwendung zu gelangen. Das Schlusskapitel dieses Teils (Kapitel 12) weist auf die problematischen Einseitigkeiten aller drei Positionen hin und plädiert für einen integrativen Ansatz, der auf der Grundlage möglichst weitreichender faktischer Information den prinzipiellen Optimismus mit praktischer Vorsicht kombiniert. Ein wichtiger Bestandteil dieser Forderung ist eine deskriptive und normative Bestimmung des Begriffs „Mensch“ im Rahmen der Debatten über mögliche Verbesserungen von Menschen. Diesem Desiderat widmet sich der entscheidende und umfangreichste Schlussteil der Arbeit.

Der vierte Teil unternimmt den Versuch, eine Methode vorzustellen und zu erproben, die es erlaubt, den Begriff „Mensch“ in deskriptiver und normativer Hinsicht so gut wie möglich inhaltlich zu bestimmen. Dazu wird als erste Voraussetzung (Kapitel 13) erläutert, wie Normativität und Begriffsbildung im außermoralischen und im moralischen Sinne zusammenhängen. Sodann wird als zweite Voraussetzung (Kapitel 14) eine Kritik von Versuchen einer direkten normativen Bestimmung des Begriffs „Mensch“ vorgenommen, die damit disqualifiziert werden. AnschlieBend wird (Kapitel 15) das Ziel einer deskriptiven und normativen Bestimmung des Begriffs „Mensch“ vorgestellt. Gemäß dem hier vertretenen moderaten oder ,,unaufgeregten“ Realismus kann das Ziel mit dem Anfertigen einer signifikanten Landkarte verglichen werden, in der deskriptive und normative Aspekte miteinander verbunden sind. Die Begründung der Normativität dieser signifikanten Karte verläuft (Kapitel 16) über einen Akt der quasi-demokratischen Verständigung, der unter den Bedingungen der Öffentlichkeit und der wechselseitigen Information nach Partizipation und Konsens unter denjenigen strebt, die sich selbst unter dem Begriff verstehen können. Dieser Verständigungsprozess ist nicht abschließbar und generiert lediglich „Zwischenergebnisse", die grundsätzlich fallibel sind und zukünftigen Revisions- 
versuchen ausgesetzt werden. An diesen methodischen Vorschlag anschließend, wird (Kapitel 17) ein Modell für die inhaltliche Bestimmung der signifikanten Kartierung vorgeschlagen. Dabei werden insbesondere vier Komponenten bestimmt, deren Struktur die Grundzüge des Begriffs im gesuchten deskriptiven und normativen Sinne erfasst. Die vier Komponenten erläutern, dass Menschen lebendig sind, aufgrund ihrer spezifischen Verkörperung und Geistigkeit ,in einer Welt" leben, darin orientierungsbedürftig und selbstbestimmungsfähig sind. Darauf aufbauend werden aus anthropologischer Perspektive zwei Bedingungen für erlaubte Veränderungen von Menschen formuliert, in deren Zentrum die Selbstbestimmungsfähigkeit des Menschen steht. Das nachfolgende Kapitel (Kapitel 18) diskutiert vier mögliche Fragen, die sich angesichts dieses (methodischen und inhaltlichen) Vorschlags einer Bestimmung des Begriffs „Mensch“ stellen können: Kann diese Bestimmung ,wahr“ sein? Ist das hier vorgeschlagene Vorgehen praktikabel oder allein eine philosophische Fiktion? Können sich nicht alle, die an der quasi-demokratischen Verständigung partizipieren, schlichtweg irren, so dass das Verfahren insgesamt unzulänglich ist? In welchem Sinne handelt es sich bei dem Vorschlag um ,anthropologische“ Diskussionen im herkömmlichen Verständnis? Die Diskussion dieser Fragen erlaubt Konkretisierungen des vorgeschlagenen Modells. Das letzte Kapitel (Kapitel 19) versucht eine exemplarische Anwendung des entwickelten normativen Begriffs vom Menschen angesichts der Möglichkeit biotechnologischer Enhancements. Anhand von drei Beispielen wird gezeigt, dass anthropologische Argumente eine untergeordnete Rolle bei der Beurteilung von Enhancements spielen, die zu Recht von den in der herrschenden Debatte diskutierten (nur indirekt anthropologisch geprägten) Risiko-, Autonomie- und Gerechtigkeitserwägungen dominiert werden. Das Schlusskapitel (Kapitel 20) fasst die Ergebnisse und Thesen der Arbeit zusammen und ordnet sie in den Kontext der Verständigung über den systematischen Zusammenhang von Anthropologie und Ethik einerseits und in das Projekt einer pragmatischen Ethik andererseits ein. 
Bereitgestellt von | UZH Hauptbibliothek / Zentralbibliothek Zürich Angemeldet Heruntergeladen am | 20.11.17 08:23 